\title{
François Sellal
}

\section{ADRESSE}

F. Sellal : docteur en médecine, directeur de l'unilé de neuropsychologie. Service de neurologie, neuropsychologie et explorations fonctionnelles des épilepsies, Hôpitaux universitaires, 67091 Strasbourg Cedex, France.

\section{TIRÉS À PART}

F. Sellal.

$m / s n^{\circ} 11$, vol. 11, novembre 95

\section{L'action amnésiante des benzodiazépines chez l'homme}

Les benzodiazépines (BZ), dont la fonction majeure est la sédation, sont susceptibles d'entraîner une amnésie antérograde massive et transitoire. Bien que la comparaison de ces deux effets soit limitée par des problèmes méthodologiques, de nombreux arguments militent en faveur d'une dissociation, au moins partielle, entre amnésie et sédation : les $\mathrm{BZ}$ ont un effet sédatif comparable à celui des barbituriques, mais elles seules sont amnésiantes ; des BZ aux structures proches peuvent entraîner une sédation similaire mais une amnésie différente ou dissociée dans le temps de l'effet sédatif : le flumazénil n'inverse pas de façon synchrone la sédation et l'amnésie induites par le diazépam et le phénomène de tolérance se manifeste plus pour la sédation que pour l'amnésie. Il semble donc que les $\mathrm{BZ}$ aient une action amnésiante propre, en partie secondaire à leur action sédative et majorée par celle-ci. Les implications pratiques et fondamentales de cette dissociation des effets sont importantes à considérer.

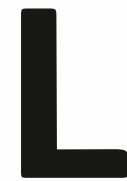

orsqu'ils synthétisèrent les premières benzodiazépines (BZ), dont la structure de base rappelait peu ou prou celle de la morphine, les pharmacologues bâlois escomptaient obtenir un antalgique. Leurs espoirs furent rapidement déçus, mais presque aussi vite réveillés par la découverte de multiples propriétés pharmacologiques inattendues. Celles-ci ont depuis lors placé les $\mathrm{BZ}$ parmi les médicaments les plus prescrits de la pharmacopée. Il s'agit en effet de molécules qui possèdent pour la plupart quatre grands effets thérapeutiques. Les deux plus connus sont l'effet tranquillisant et l'effet sédatif-hypnotique; il faut y ajouter un effet anti- épileptique remarquable, ainsi qu'un effet myorelaxant (décontracturant). A cheval entre les effets thérapeutiques et indésirables figure une action singulière, qui est l'action amnésiante des BZ. Celle-ci a d'abord été notée par les anesthésistes utilisant les BZ comme tranquillisants avant l'acte opératoire: les malades qui avaient reçu une $\mathrm{BZ}$ avaient beaucoup plus de mal à se rappeler la journée de l'opération que ceux ayant reçu un barbiturique ou un placebo [1]. La double propriété amnésiante et tranquillisante des $\mathrm{BZ}$ leur conférait un net avantage sur tous les médicaments jusqu'ici proposés comme préanesthésiques, qui n'avaient que l'une ou l'autre action [2]. 


\section{RÉFÉRENCES}

1. Brandt AL, Oakes FD. Preanesthesia me dication: double-blind study of a new drug, diazepam. Anesth Analg 1965 ; 44 : 125-9.

2. Pandit SK, Dundee IW. Preoperative amnesia: the incidence following the intramuscular injection of commonly used premedicants. Anaesthesia 1970; 25 : 493-9.

3. Lister RG. The amnesic action of benzodiazepines in man. Neurosi Behav Rev 1985 9: 87-9.

4. Ghoneim MM, Hinrichs JV, Mewaldt SP. Dose-response analysis of the behavioral ef fects of diazepam: 1. Learning and memory. Psychopharmacology 1984; 82: 291-5.

5. Sellal F, Bacon E, Collard M. Mémoire et benzodiazépines. Rev Neurol (Paris) 1994 $150: 330-7$.

6. Hinrichs JV, Mewaldt ST, Ghoneim MM, Berie JL. Diazepam and learning: assessment of acquisition deficits. Pharmacol Biochem Behav 1982; 17: 165-70.

7. Brown J, Lewis V, Brown MW, Horn G, Bowes JB. A comparison between transient amnesias induced by two drugs (diazepam and lorazepam) and amnesia of organic origin. Neuropsychologia 1982; $20: 55-70$.

8. Preston GC, Broks P, Traub M, Ward C, Poppleton P, Stahl SM. Effects of lorazepam on memory, attention and sedation in man Psychopharmarology 1988; 95: 208-15.

9. Sellal F, Danion JM, Kauffmann-Muller F, et al. Differential effects of diazepam and lorazepam on repetition priming in healthy volunteers. Psychopharmacology 1992; 108 : $371-9$.

10. Roth T, Hartse KM, Saab PG, Piccione PM, Kramer M. The effects of flurazepam, lorazepam, and triazolam on sleep and memory. Psychopharmacology 1980; 70: 231-7.

11. File SE, Lister RG. Does tolerance to lorazepam develop with once weekly dosing ? BrJ Clin Pharmacol 1982; 16: 645-50.

12. Ghoneim MM, Mewaldt SP, Berie JL, Hinrichs JV. Memory and performance effects of single and 3-week administration of diazepam. Psychopharmacology $1981 ; 73: 147$ 51

13. Roache JD, Griffiths RR. Comparison of triazolam and pentobarbital: performance impairment, subjective effects and abuse liability. J Pharmacol Exp Ther 1985; $224: 120$ 33.

14. Curran VH. Benzodiazepines, memory and mood: a review. Psychopharmacology $1991 ; 105: 1-8$.

15. Curran HV, Schiwy W, Lader M. Differential amnesic properties of benzodiazepines: a dose-response comparison of two drugs with similar elimination half-lifes. Psy-
L'action amnésiante des BZ s'observe avant tout chez le consommateur inhabituel de $\mathrm{BZ}$, après la prise d'une dose unique et suffisamment importante du médicament. Cette amnésie peut être massive mais elle est toujours transitoire, le temps que l'effet du médicament s'estompe. Elle n'empêche pas la poursuite d'une activité parfaitement coordonnée, dans la mesure où la sédation n'est pas trop marquée et où cette activité ne nécessite pas l'utilisation d'informations apprises sous l'emprise du médicament [3]. La BZ entraîne, en effet, une amnésie pour les faits survenus après la prise du médicament. Elle réalise donc ce qu'on appelle une amnésie antérograde, caractérisée par un oubli à mesure des événements survenus pendant qu'agit le médicament. Le mécanisme de cette amnésie a été mieux précisé par des études comme celle de Ghoneim et al. [4]. On y a demandé à des volontaires sains d'apprendre quatre listes de mots, l'une avant toute prise de médicament, les autres à intervalle régulier après prise de diazépam ou de placebo. Une heure trente après l'ingestion, le rappel différé des mots appris sous l'emprise du médicament était sévèrement altéré chez les sujets ayant reçu le diazépam. En revanche, le rappel des mots de la liste apprise avant ingestion de diazépam était préservé. Cela suggère deux conclusions: d'une part, le stockage et le rappel des informations anciennes (c'est-à-dire apprises avant la prise de BZ) ne sont pas altérés par les $\mathrm{BZ}$; d'autre part, l'absence de mémorisation des informations nouvelles (donc présentées sous l'emprise de la $\mathrm{BZ}$ ) résulte d'une perturbation de leur acquisition sous BZ.

Un tel profil des troubles de la mémoire est très proche de celui observé dans des amnésies organiques, telles que l'ictus amnésique ou le syndrome de Korsakoff. Ce dernier, le plus souvent observé chez de grands alcooliques, est dû à une carence en vitamine $B_{1}$, qui entraîne des lésions irréversibles sur les circuits de la mémorisation. Or, on sait que dans de telles amnésies organiques certaines capacités de mémorisation, telles que la mémoire rétrograde (mémoire des faits anciens), sont préservées. De très nombreuses études menées ces vingt dernières années ont montré que l'amnésie due aux $\mathrm{BZ}$ et des amnésies organiques telles que le syndrome de Korsakoff partageaient de façon assez fidèle le même profil ( $T a$ bleau l): les formes de mémoire altérées ou préservées sont en effet très superposables dans les deux sortes d'amnésie (revue dans [5]).

La constatation d'une telle action amnésiante des $\mathrm{BZ}$ a suscité de nombreuses recherches, avec deux grandes orientations: une meilleure connaissance de cette action afin de mieux la prévenir en clinique, lorsqu'elle peut être indésirable chez le sujet traité; une utilisation de ce modèle d'amnésie chez le sujet sain, afin de mieux comprendre le fonctionnement de la mémoire normale. En neuropsychologie, il est, en effet, classique d'analyser les déficits partiels d'une fonction (le plus souvent induits par une maladie: accident vasculaire cérébral, traumatisme crânien, etc.), afin de valider ou d'infirmer des hypothèses faites sur les processus sous-tendant cette fonction. Ainsi, l'étude de la mémoire doit beaucoup à des patients présentant un syndrome de Korsakoff. Un des principaux écueils d'une telle approche de la mémoire est qu'il est souvent difficile de trouver des malades souffrant de cette maladie et que leur tableau clinique est rarement le même. Or, les BZ, données dans certaines conditions précises, peuvent induire un syndrome amnésique relativement homogène. Les neuropsychologues ont ainsi trouvé un modèle d'amnésie présentant de multiples avantages sur les amnésies observées en médecine: elle est très reproductible et dépend de la dose; elle peut être induite "à la demande " chez des volontaires sains (qui auront pour toute séquelle un trou de mémoire de quelques heures); elle est transitoire et permet donc de tester l'efficience mnésique du sujet avant, pendant, voire après l'amnésie, de façon à avoir des performances de référence [5].

\section{Spécificité de l'effet amnésiant}

Dans l'une et l'autre approche de recherche, clinique ou fondamentale, un préalable important a été de savoir dans quelle mesure l'action 


\section{Tableau I}

TROUBLES COMPARÉS DE LA MÉMOIRE DANS LE SYNDROME DE KORSAKOFF ET L'AMNÉSIE INDUITE PAR LE DIAZÉPAM

\begin{tabular}{|c|c|c|}
\hline Formes de mémoire & $\begin{array}{l}\text { Syndrome } \\
\text { de Korsakoff }\end{array}$ & $\begin{array}{c}\text { Amnésie } \\
\text { due au diazépam }\end{array}$ \\
\hline $\begin{array}{l}\text { Mémoire rétrograde } \\
\text { (faits anciens) }\end{array}$ & normale & normale \\
\hline $\begin{array}{l}\text { Mémoire antérograde } \\
\text { (faits nouveaux) }\end{array}$ & altérée & altérée \\
\hline Mémoire à court terme & normale & normale \\
\hline $\begin{array}{l}\text { Mémoire à long terme } \\
\text { - épisodique (factuelle) } \\
\text { - sémantique } \\
\text { (connaissances générales) } \\
\text { - procédurale } \\
\text { (façon de faire) } \\
\text { - amorçage }\end{array}$ & $\begin{array}{l}\text { altérée } \\
\text { normale } \\
\text { normale } \\
\text { normal }\end{array}$ & $\begin{array}{l}\text { altérée } \\
\text { normale } \\
\text { normale } \\
\text { normal }\end{array}$ \\
\hline Conscience de l'amnésie & altérée & altérée \\
\hline
\end{tabular}

(1) On désigne par amorçage la facilitation du traitement d'un stimulus $S$, due au traitement préalable de $S$ ou d'un stimulus sémantiquement proche.

amnésiante des BZ était spécifique et non pas secondaire à l'un des autres effets des BZ. En effet, comme ces médicaments sont sédatifs, il était possible que l'amnésie ne fût que la conséquence d'une baisse du niveau de vigilance.

Les difficultés à quantifier la sédation ou à mesurer le niveau attentionnel contribuent à compliquer la question. Ainsi, divers facteurs peuvent perturber la composante motrice de tests psycho-moteurs censés mesurer la sédation. Par exemple, certaines épreuves graphiques (telles que des épreuves de transcodage de symboles), fréquemment utilisées comme index de sédation, peuvent être perturbées par l'incoordination motrice induite par les BZ. Dans un grand nombre d'études on a eu recours à des échelles d'auto-évaluation de la sédation. Or, cela postule que le sujet soit capable d'apprécier son niveau de vigilance, ce qui n'est pas certain [6]. Enfin, de nombreux index classiques de sédation manquent de sensibilité: temps de réaction simple, vitesse de tapotement d'un doigt, empan des chiffres [7-9]; la mesure d'une latence d'endormissement [10] n'évalue que la vigilance $a$ posteriori et non les capacités d'attention sélective. Par conséquent, toute dissociation apparente entre les effets des BZ sur la mémoire et sur l'attention pourrait ne traduire que des différences de sensibilité des moyens de mesures de l'une et l'autre. Une fois ces limitations posées, il persiste un faisceau d'arguments, de valeur inégale, suggérant, malgré tout, une dissociation entre effets amnésiant et sédatif.

\section{Analyses statistiques}

Leurs résultats sont discordants. Dans une étude évaluant l'importance de la récapitulation, de la motivation et de la sédation sur l'effet amnésiant du lorazépam, File et Lister [11] avaient observé l'existence d'une corrélation entre les auto-évaluations de la sédation et les troubles mnésiques. Cependant, cette corrélation entre amnésie et sédation n'a pas été retrouvée par tous les chercheurs (par exemple, [12, 13]). D’après les analyses de Curran [14, 15], une fois égalisé le niveau de sédation entre les BZ, celles-ci gardent toutes un effet amnésiant, moindre mais significatif par rapport au placebo. D'après ces données, la sédation contribuerait mais ne suffirait pas à expliquer l'amnésie induite par les BZ.

\section{Dissociation selon la molécule}

Plusieurs auteurs ont fait remarquer qu'il pouvait y avoir une dissociation expérimentale entre les effets sédatifs et amnésiants: ainsi, on sait que des produits ayant un effet sédatif, tels que les opiacés ou les butyrophénones, n'ont pas ou ont peu d'effet amnésiant $[16,17]$. Si l'on compare les actions du triazolam, une $\mathrm{BZ}$, et du pentobarbital, un barbiturique, on observe que le triazolam a un effet amnésiant bien plus marqué que le pentobarbital, à sédation égale [13]. Les diverses $\mathrm{BZ}$ elles-mêmes ne se comportent pas de façon homogène. Ainsi, deux BZ chimiquement proches, l'oxazepam et son dérivé chloré, le lorazepam entraînent une altération similaire des marqueurs de l'attention, de la sédation et des habiletés psychomotrices, tandis que leurs effets sur la mémoire sont différents [15].

\section{Dissociation selon la dose}

Les effets sédatif et amnésiant peuvent également se manifester de façon dissociée selon la dose administrée. Ainsi, l'effet amnésiant du flunitrazépam apparaît dès les faibles doses (1 mg), alors qu'une altération des mesures de l'attention ne se manifeste qu'à des doses plus élevées (2 ou $4 \mathrm{mg}$ ) [18]. Dans un même ordre d'idées, $5 \mathrm{mg}$ de diazépam préservent la mémoire à court terme (sensée être un témoin des capacités d'attention), mais altèrent la mémoire à long terme [19].

\section{Dissociation dans le temps}

Dans quelques études on a utilisé un antagoniste des BZ, le flumazénil, qui entraine une disparition rapide des effets des BZ agonistes. Hommer et al. [20] ont ainsi administré du flumazénil préalablement à un agoniste, le diazépam. Les effets sédatifs du diazépam furent bloqués mais un effet amnésiant marqué se manifesta. D'autres auteurs montrèrent que le flumazénil supprimait les effets amnésiant et sédatif du diazépam [21] ou du midazolam [22], mais pas de façon concomitante. Ces résultats, pas tout à fait concordants, permettent au moins d'avancer l'hypothèse selon laquelle les effets sédatifs 


\section{RÉFÉRENCES}

16. Clark CR, Geffen GM, Geffen LB. Role of monoamine pathways in the control of attention. Effects of droperidol and methylphenidate in normal adult humans. Psycho pharmacology $1986 ; 90: 28-34$

17. Scamman FL, Ghoneim MM, Kortilla K Ventilatory and mental effects of alfentany and fentanyl. Acta Anaesthesiol Scand 1984 28: $63-7$.

18. Smirne S, Ferini-Strambi L, Pirola R, Tancredi $O$. Effects of flunitrazepam on cognitive functions. Psychopharmacology 1989 98: 251-6.

19. Lucki I, Rickels K, Geller AM. Chronic use of benzodiazepines and psychomotor and cognitive test performance. Psychopharmacology $1986 ; 88$ : 426-33.

20. Hommer DW, Weingartner $\mathrm{H}$, Breier $\mathrm{A}$. Dissociation of benzodiazepine-induced amnesia from sedation by flumazenil pretreat ment. Psychopharmacology 1986; 112: 455-60.

21. Ghoneim M, Dembo J, Block R. Time course of antagonism of sedative and amnesic effects of diazepam by flumazenil. Anesthesiology 1989; $70: 899-904$.

22. Curran VH, Birch B. Differentiating the sedative, psychomotor and amnesic effects of benzodiazepines: a study with midazolam and the benzodiazepine antagonist, flumazenil. Psychopharmacology 1991 ; 103: 519-23.

23. Morton S, Lader MH. Studies with alpidem in normal volunteers and anxious patients. Pharmacopsychiatry $1989 ; 23: 120-3$

24. Danion JM, Sellal F. Le médicament, un outil d'investigation des fonctions cognitives. Ie Courrier du Conrs 1992; 79 : 61 .

25. Izquierdo I, Medina JH. GABA - A receptor modulation of memory: the role of endogenous benzodiazepines. Trends Pharmacol Si $i$ 1991; 12: 260-5.

26. Unseld E, Krishna DR, Fischer C, Klotz U. Endogenous benzodiazepines in brain: right or wrong ? Trends Neurosai 1988; 11 : 490 .

27. Chapouthier G, Raffalli-Sébille M. Du nouveau par l'étude des agonistes partiels du récepteur des benzodiazépines. médecine/sciences $1989 ; 5$ : 504-6.

28. Roehrs T, Merlotti L, Zorick F, Roth T. Sedative, memory, and performance effects of hypnotics. Psychopharmacology 1994; 116 : $130-4$.

29. Bacon $\mathrm{E}$, Viennot $\mathrm{F}$. Le système complexe des récepteurs GABA-benzodiazépine. médecine/sciences $1990 ; 6: 770-7$.

30. Doble A, Martin IL. Multiple benzodiazepine receptors: no reason for anxiety. Trends Neurosci $1992 ; 131$ : 76-81.

31. Chapouthier G, Martin B. Beta-carbolines: from memory towards genetics. Eur des $\mathrm{BZ}$ sont plus facilement contrés que leur action amnésiante.

Le fait que la tolérance pour l'effet amnésiant et sédatif des $\mathrm{BZ}$ n'apparaisse pas de façon concomitante cons titue un argument supplémen taire en faveur d'une dissociation. On désigne par tolérance le fait que pour continuer à obtenir un même effet pharmacologique, il faille parfois augmenter la dose du médicament au fil du temps. Or, la survenue d'une tolérance est plus rapide pour l'effet sédatif ou psychomoteur que pour l'effet amnésiant. Ainsi, il persiste une altération du rappel libre après qua tre semaines de traitement par lorazépam ou après huit semaines de trai tement par alprazolam, alors que la sédation s'est déjà nettement amenuisée [12, 23].

\section{Perspectives}

En résumé, il semble que, si la sédation peut expliquer, pour une part, l'effet amnésiant des BZ, elle n'en constitue pas le seul déterminant. Les $\mathrm{BZ}$ auraient donc un effet amnésiant propre. Le corrolaire est qu'il y a théoriquement possibilité de dissocier les effets amnésiant et sédatif.

Dans le domaine de la recherche fondamentale, cela constitue une validation du modèle d'amnésie due aux $\mathrm{BZ}$. Ces molécules peuvent même devenir une sorte de "scalpel pharmacologique", permettant de disséquer la mémoire normale en perturbant électivement certaines de ses formes [24]. En outre, elles off rent une passionnante ouverture sur la neurobiologie de la mémoire. Au niveau de la membrane neuronale, on sait que les $\mathrm{BZ}$ se fixent sur les récepteurs $\mathrm{GABA}_{\mathrm{A}}$, qui prédominent dans le cortex cérébral et le cervelet. C'est leur abondance dans l'hippocampe qui expliquerait l'action amnésiante des BZ [25]. L'investigation du rôle exact de ces récepteurs et de leurs ligands naturels dans les processus d'apprentissage et de mémoire, leur étude in vivo à l'aide de la tomographie à émission de positons constituent un champ de recherche prometteur [26]. Par exemple, chez l'homme, assez peu de choses sont à ce jour connues sur la façon dont les BZ diffèrent entre elles in vivo dans leur fixation cérébrale ou leur affinité pour certains récepteurs $\mathrm{GABA}_{\mathrm{A}}$.
Dans le domaine clinique, les plus récentes évaluations d'hypnotiques tendent à mieux prendre en compte l'effet amnésiant, parfois en imaginant des tests plus écologiques que les tests de laboratoire. Ainsi, dans certaines études, les sujets devaient retenir un ensemble d'événements vécus avant le coucher et les rappeler après une nuit sous BZ [10]. Par ailleurs, on peut espérer synthétiser un jour des BZ mieux ciblées. On sait en effet que le récepteur $\mathrm{GABA}_{A}$ comporte divers sous-types dont la distribution cérébrale diffère et qui ont probablement des rôles physiologiques distincts. Une voie prometteuse d'avenir s'entrouvre avec la découverte des premiers agonis tes partiels. Con trairement aux $\mathrm{BZ}$ agonistes classiques, qui ont les cinq actions mentionnées en in troduction, ces autres BZ, du fait de leur plus grande affinité pour certains sous-types de récepteurs $\mathrm{GABA}_{\mathrm{A}}$, n'ont qu'une partie de ces actions. A la fin des années 1980 la description de récepteurs dits $B Z_{1}$ et $B Z_{2}$ avait fait espérer la synthèse de produits ayant l'action sédative-hypnotique et peu d'action amnésiante. En effet, ces récepteurs se distribuent de façon équivalente dans le cervelet, le cortex cérébral et la substance noire, mais les récepteurs $\mathrm{BZ}_{2}$ sont bien plus représen tés dans l'hippocampe (structure clé dans les phénomènes de consolidation de la trace mnésique) [27]. Or on dispose désormais de molécules spécifiques des récepteurs $\mathrm{BZ}_{1}$, les imidazopyridines. Des études ultérieures menées chez l'homme ont cependant montré que ces molécules, bien que se fixant peu sur les récepteurs hippocampiques, gardent un effet amnésiant [28]. L'avenir se situe probablement dans une connaissance encore plus poussée des sous-types de récepteurs [29]. A ce jour, la dichotomie en $\mathrm{BZ}_{1}$ et $\mathrm{BZ}_{2}$ est déjà devenue obsolète. Pour une seule des sous-unités du récepteur, dite $\alpha$, on connaît désormais six isof ormes différentes, pour lesquelles les $\mathrm{BZ}$ ou imidazopyridines ont une affinité con trastée $(\mathrm{m} / \mathrm{s}$ $n^{\circ} 3$, vol. 9, p. 342) [30].

Une autre ouverture vient de la découver te de molécules dites agonistes inverses. Ces molécules, qui se fixent aussi sur le récepteur $\mathrm{GABA}_{A}$, ont des effets exactement inverses des $\mathrm{BZ}$ agonistes. C'est le cas des $\beta$-carbolines, qui sont donc promnésiantes, 
mais malheureusement inutilisables car anxiogènes et épileptogènes [31] . Il n'est cependant pas exclu que, là aussi, on trouve un jour des molécules mieux ciblées, n'ayant plus que l'effet promnésiant.

Toute cette approche, fondamentale et clinique, s'inscrit dans un courant de recherche plus vaste de la "psychopharmacologie ", qui cherche à mieux comprendre le rôle des différents neuromédiateurs dans la pensée et le comportement, normaux ou pathologiques. Cette discipline passe par une meilleure connaissance des récepteurs et neuromédiateurs, mais beaucoup de chemin reste encore à parcourir avant de savoir corréler, chez l'homme, la fixation d'une molécule à un sous-type de récepteur précis et un effet comportemental donné

\section{Summary}

The amnesic action of benzodiazepines in man

Benzodiazepines (BZs) may induce a massive but transient amnesia: they affect the acquisition of new information, whereas retrieval of already learned information is unimpaired. As BZs can also be sedative, there is a question as to whether the amnesic effect is independent from the sedative effect. Although the comparison between these two effects depends on the methods used, there may well be at least a partial dissociation between amnesia and sedation: i) although BZs have the same sedative effect as barbiturates, only the BZs have an amnesic effect; ii) BZs as similar as oxazepam and lorazepam can lead to a similar sedation but a different amnesia; iii) the amnesic effect can be dissociated temporally from the sedative effect; iv) flumazenil antagonises but not synchronously the sedation and amnesia induced by diazepam; $v$ ) the tolerance for sedation is greater than that for amnesia. Thus BZs appear to have a specific amnesic effect, not uniquely secondary to their sedative powers, but increased by them. Practical and fundamental implications of this dissociation of effects are to be considered. 